\title{
The Application of Advanced Technique of Fan Frame Unit on High Bypass Ratio Aero Engine
}

\author{
HOU Peng ${ }^{1, a}$, LUO ai-ran ${ }^{1}$ and HAN fang-jun ${ }^{1}$ \\ ${ }^{1}$ Shenyang Aero Engine Design and Research Institute, 2 Department, Shenyang, China
}

\begin{abstract}
High bypass ratio aero-engine was widely used on military and civil aviation domain, as the power of larger aircraft. Fan frame unit was the main bearing frame of high bypass ratio aero-engine, which composed of strut, HUB MID BOX and external bypass parts. Resin/composite was used on external bypass parts(acoustic liner, containment ring, fan outlet guide vane and fan case skin fillets), which not only reduced the weight and manufacturing cost, but also improved the noise absorption, containment and anti-fatigue ability of engine. The design of composite was becoming a key technique for high bypass ratio aero-engine. In special test of the core engine, nitrogen cooling system was designed to cool the cavity of spool. The nitrogen pipeline passed through the inner cavity of fan frame, then inserted into NO. 3 bearing seal, so nitrogen gas was sent into the cavity of core engine spool. On high bypass ratio aero-engine, the external bypass and fan frame inner cavity were the design platform for advanced technique, such as composite and pipeline system, and also provided guarantee for reliable operation of engine.
\end{abstract}

\section{Introduction}

If the bypass ratio of turbofan aero-engine was greater than 4, it was called high bypass ratio aeroengine. The advance of high bypass ratio aero-engine was larger thrust, lower noise, better safety and good reliability. High bypass ratio aero-engine was the power plant of large military and civil aircraft. Fan frame unit was the main bearing frame of high bypass ratio aero engine, which composed of 12 struts, HUB MID BOX and external bypass parts. The strut cavity and HUB MID BOX cavity was communicated with each other, providing channel for oil pipe, air pipe, testing cable and transmission rod. The fan frame cavity of high bypass ratio aero-engine was relatively bigger, so more pipeline could be routed, such as nitrogen cooling system, as shown of figure $1(7,8)$. In order to meet the requirement of higher thrust-weight ratio and lower maintenance cost, composite was widely used on high bypass ratio aero-engine [1]. Compared with titanium alloy, composite had the advantage of lighter weight, higher specific strength, higher specific stiffness, and better damping ability. On high bypass ratio aero-engine, the external bypass parts (acoustic liner, containment ring, fan outlet guide vane and fan case skin fillets) were all used composite as shown of figure 1(1 6), which contained resin glass fiber composite, resin carbon fiber composite and resin Kevlar fiber composite. In ground and flight test, the composite showed excellent performance. The design of composite was becoming a key technique for high bypass ratio aero-engine.

\footnotetext{
${ }^{a}$ HOU Peng : houpeng2749@sina.com
} 


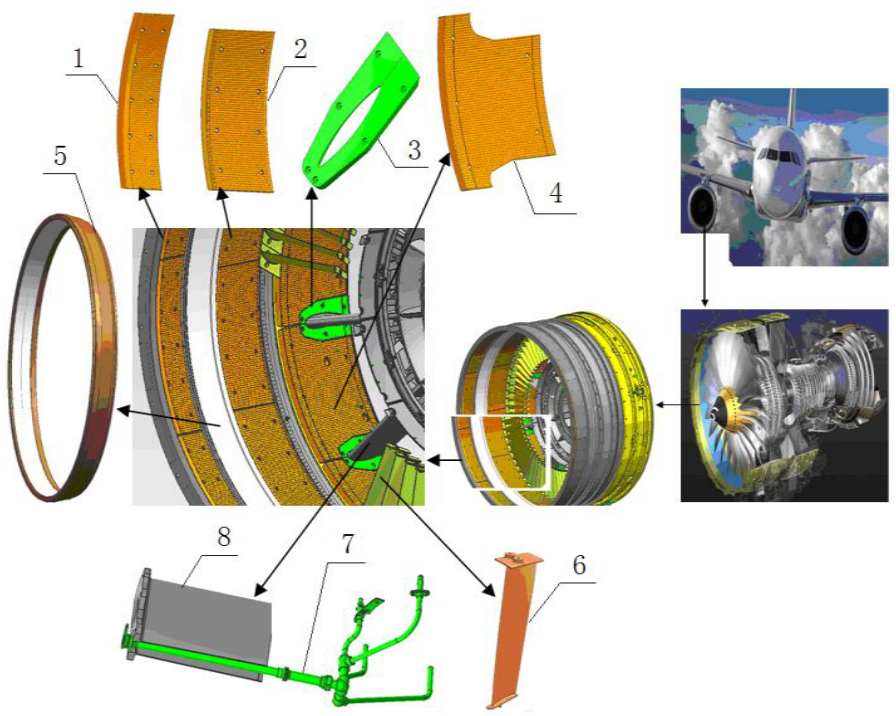

Figure 1. Explosive view of fan frame

1-Acoustic liner, 2-Acoustic liner, 3-Fan case skin fillets, 4-Acoustic liner, 5-Containment ring, 6- Fan outlet guide vane, 7-Pipeline system, 8- Strut

\section{Composite Acoustic Liners}

Fan was the main noise source of aero engine, and reducing fan noise was the primary task of engine noise reduction [2]. At present, the main method to reduce fan noise was using acoustic liner, and the acoustic liners were assembled on external bypass (secondary airflow bypass), as shown of figure 1(1, 2, 4). A good simple model for an acoustic liner is the Helmholtz resonator, and the acoustic liner model begins with a simplified acoustic system and then is advanced by building it up to the more complicated liner system [3], as shown of figure 2(b).

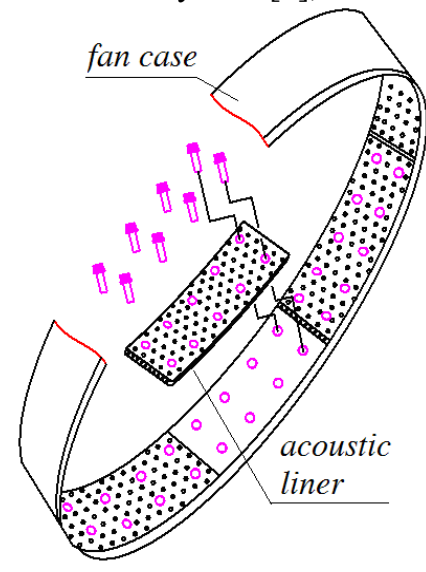

(a)Assembly view

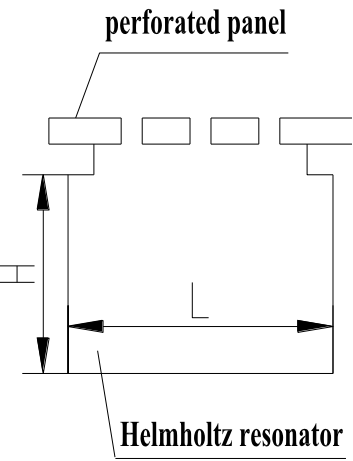

(b) Helmholtz resonator

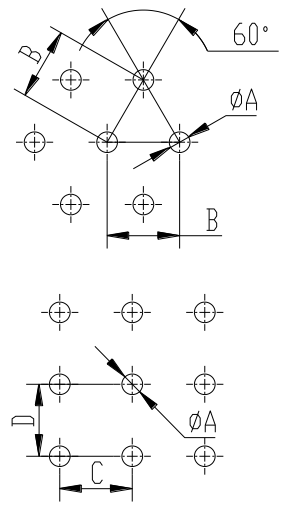

(c) Perforation ratio

Figure 2. Assembly and structure of segment acoustic liner

The noise absorption of acoustic liner was determined by the parameter as follow: bore perforation ratio of panel, material of honeycomb, and the dimension of honeycomb core $[4,5]$. The rank of bores on perforated panel were regular triangle and rectangular array, the perforation ratio formula were as follow. 


$$
\begin{aligned}
& \sigma=\frac{\pi \cdot A^{2}}{2 \sqrt{3} B^{2}-\pi \cdot A^{2}} \\
& \sigma=\frac{\pi \cdot A^{2}}{4 C \cdot D-\pi \cdot A^{2}}
\end{aligned}
$$

"A" represented bore diameter, "B", "C", "D" represented bore distance, and " $\sigma$ "represented the perforation rate of perforated panel. Formula (1) was the perforation rate for regular triangle array bores, and formula (2) was the perforation rate for rectangular array bores, as shown of figure 2(c). As fan mainly produced shock wave noise, the bores of fan case acoustic liner were regular triangle array, and perforation rate was $7.4 \%$. High frequency noise was mainly produced around fan frame shroud, the bores of fan frame acoustic liner were rectangular array, and perforation rate was $14 \%$. For single aero engine, the noise absorption of the whole acoustic liners was $8 \sim 10 \mathrm{~dB}$.

For improving the property of noise absorption or adjusting the frequency range of noise reduction, no need to change the structure of acoustic liner and merely change the parameter (perforation rate, perforation mode, dimension and thickness of honeycomb core) concerned. Owning to the excellent noise absorption property, better vibration damping ability and stable structure of composited acoustic liner, it could be popularized on high bypass ratio aero engine.

\subsection{Segment acoustic liner}

Acoustic liner had a honeycomb panel (aramid fiber or other absorber material), a perforated panel (epoxy resin/aramid fiber), and metal bushings. The perforated panel was a cover body on the surface of honeycomb panel, and metal bushings were inserted in the bolt hole, as shown of figure 3 . Compared with metal perforated sheet, composite acoustic liner could reduce $10 \mathrm{~kg}$ for single engine.

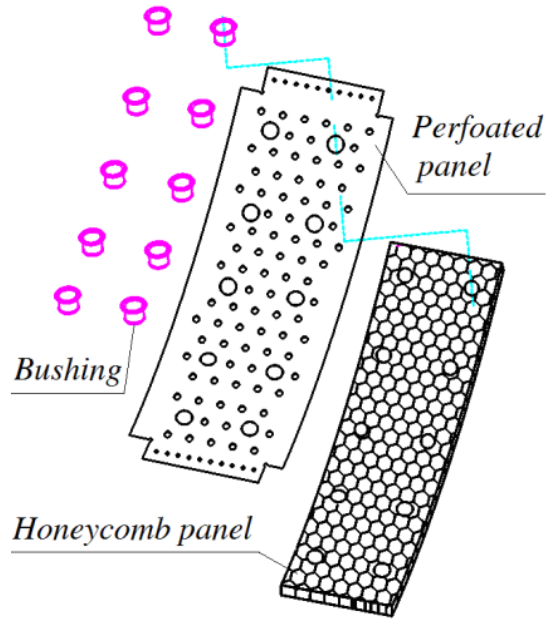

(a) Explosive view

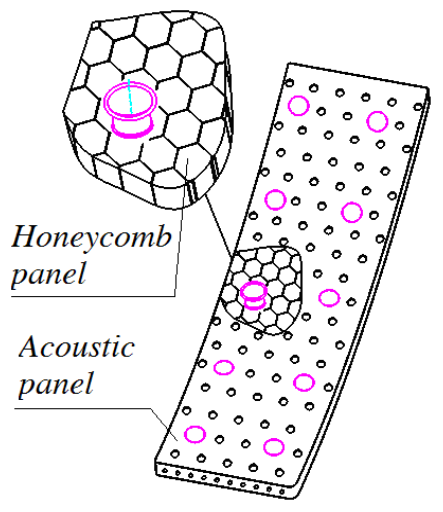

(b) Acoustic liner

Figure 3. Structure of segment acoustic liner

The process of assembly is that jointing the segment liners together on fan case and fan frame shroud, fixing the liners with bolts, and sealing the gap of joint with sealant, as shown of figure2(a).

\subsection{Integral acoustic liner}

The integral acoustic liner had a circle honeycomb panel and a circle perforated panel, which was inserted on the inner wall of fan case, as shown of figure 4. The processing technology, assembly technology and adhesion technology of integral liner is relatively strict, thereby the cost is higher. The inner structure of integral liner was the same to segment liner. Compared with integral liner, the noise 
reduction of segment liner is lower due to the acoustic scatter from the joint. The disadvantage was overcome by the integral liner, which improves the noise absorption ratio. In order to enhance the noise reduction level of aero engine, segment liner would be gradually replaced by integral liner.

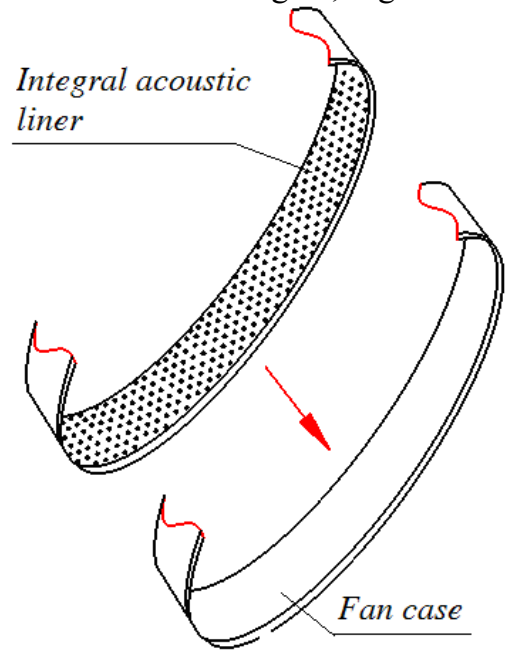

(a) Explosive view of integral liner

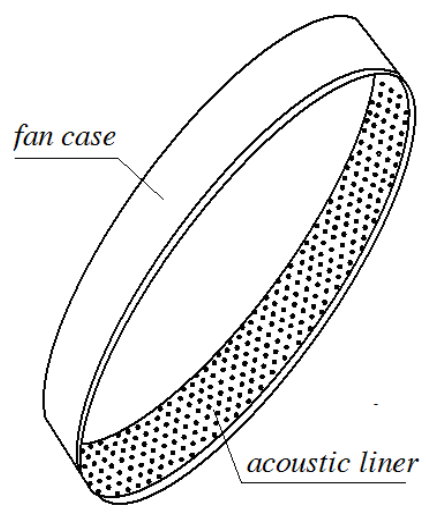

(b) Assembly view

Figure 4. Structure of integral acoustic liner

\section{Composite Skin Fillets}

Fan case skin fillets were used for filling the gap between fan frame acoustic liner and strut, and the material was glass fiber reinforced polyether composite. The skin fillet had a lighter weight, better toughness, better anti-fatigue property, and could be repeatedly disassembled and assembled, as shown of figure 1(3). The skin fillet was shell structure and the inner side was reinforced rib and installation base, and the outer side was flow path, as shown of figure 5 .

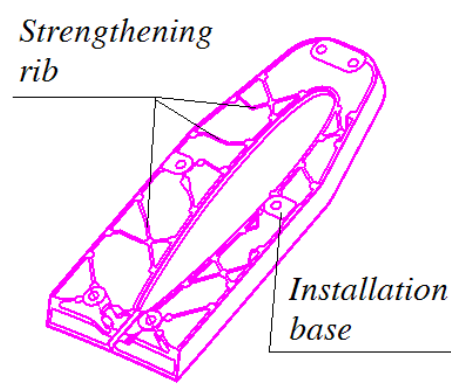

(a) Inner side

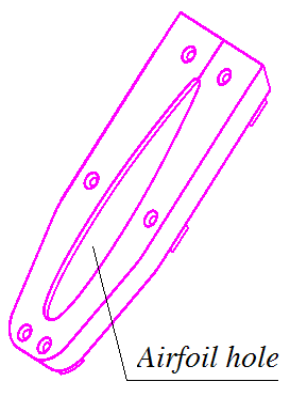

(b) Outer side

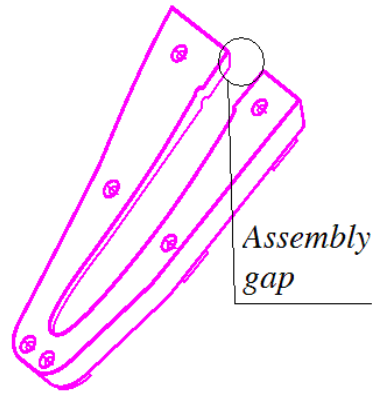

(c) Open structure

Figure 5. Structure of fan case skin fillet

Compared with metal material, composite skin fillets could reduce $5 \sim 8 \mathrm{~kg}$ for single engine. 


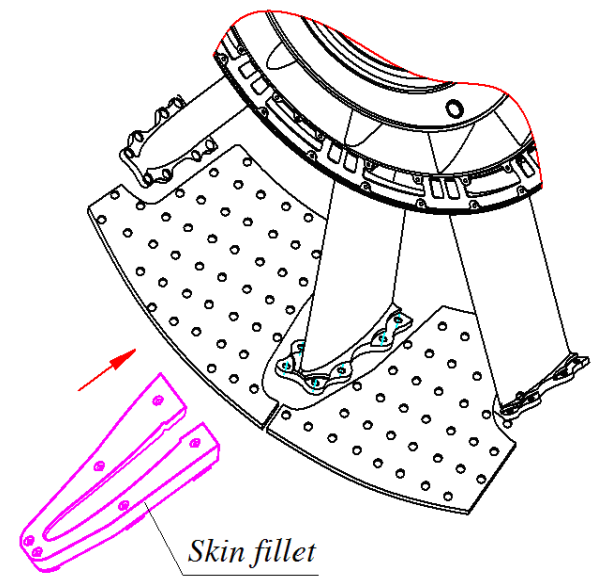

(a) Before assembly

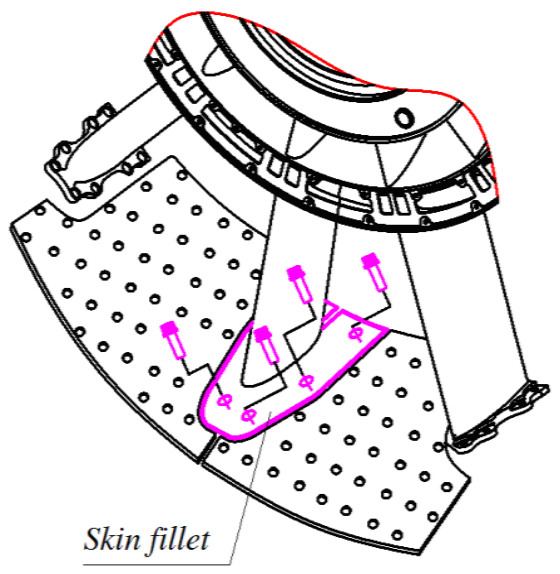

(b) After assembly

Figure 6. assembly of fan case skin fillet

Assembly method: opening the skin fillet along airfoil hole, as shown of figure5(c), and then inserting it into strut, finally fixing the skin fillet with bolts, as shown of figure 6 .

\section{Composite Outlet Guide Vane}

Fan outlet guide vanes were the bearing parts of fan frame, adjusting the secondary airflow, as shown of figure 1(6). The vane was made of epoxy resin/carbon fiber composite, and reduced $25 \sim 40 \mathrm{~kg}$ for single engine. The composite vane had higher natural frequency, better damping property, better antifatigue and excellent anti-crack ability. For overcoming the weakness of bad anti-impact and easy to delaminate, the nickel overlapping was pasted on leading edge, and the metal bushings were inserted in bolt hole, as shown in figure 7(a).
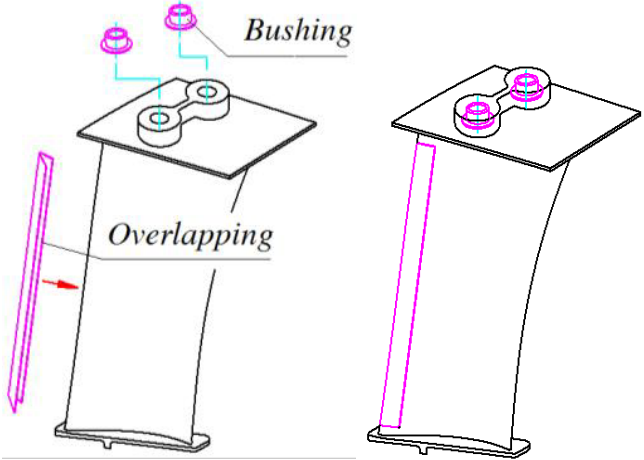

(a) Explosive view

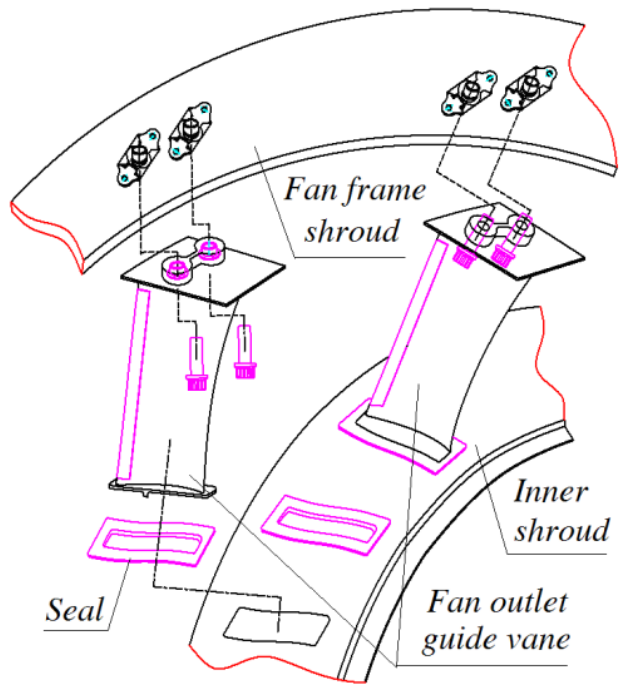

(b) Assembly view

Figure 7. Structure and assembly of fan outlet guide vane

The assembly process was as follows: firstly, inserting the inner platform into seal, then, fixing the outer platform to fan frame shroud with bolts, finally, shroud and vanes formed cantilever structure, as shown of figure 7(b). 


\section{Containment Case}

If the titanium alloy fan blade broken off from root, the centrifugal force was more than 40t. In order to prevent the released blade puncturing fan case and ensure the safety of aircraft and passengers, the fan case needed containment structure [6]. Compared with metal containment case, composite containment case could reduce $30 \sim 50 \mathrm{~kg}$ for single engine.

\subsection{Containment ring}

The containment ring was formed by winding Kevlar fiber strip, and the inner could be clamped with metal layer to enhance containment ability. The containment liner was inserted into fan case, for preventing radical vibration and deformation, the metal protect ring was fixed on the edge, as shown of figure 1(5). It had a machined groove on inner side, and the groove was coated with talc coating, and the protective coating was coated on both sides of containment liner, after talc coating being completely solidified, as shown in figure 8 . Talc coating was blended by carbonate, adhesive and epoxy resin. Through scraping talc coating, fan blade adjusted tip clearance with coating.

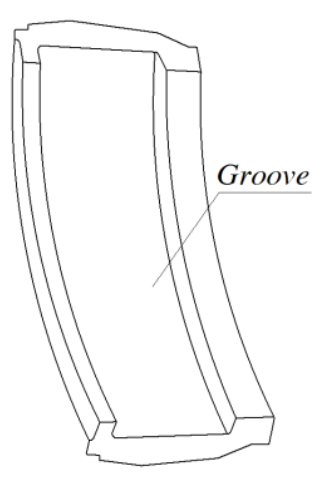

(a) Composited frame

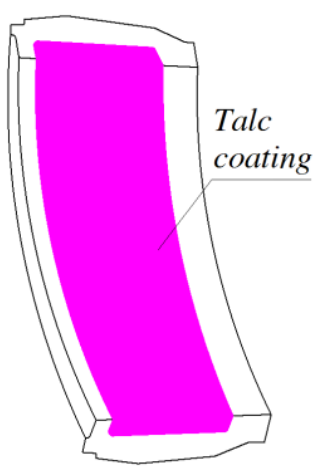

(b) Talc coating

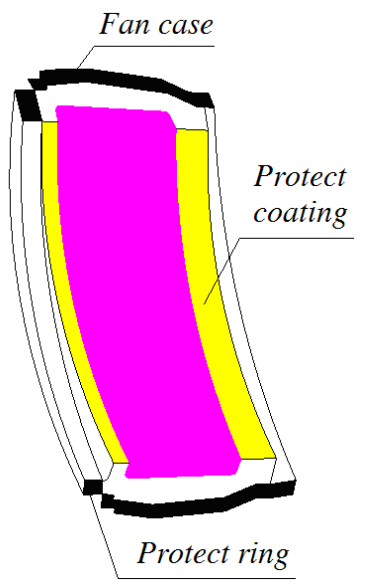

(c) Assembly

Figure 8. Structure of containment ring

After machining, the outer structure of containment would be destroyed, thereby reducing the containment ability. Three-dimensional weaving technology can avoid mechanical damage to the fiber structure, due to the high cost and technical difficulty, it is too difficult to popularize in a short time.

\subsection{Containment layer}

The metal fan case is gradually replaced by composite case, and the containment layer was winded around the outer surface of fan case, so as to improve the containment ability. The containment layer was made of carbon fiber or aramid fiber mixed with epoxy resin, and the fiber layer could absorb the impact energy of released blade, as shown in figure 9. Using finite element model with the parameter of fan blade diameter, rotated speed, and the strength of fiber, the amount of fiber layer could be got [7]. Compared with inner containment ring, the containment layer had a simple processing, simplified the structure of fan case, and enhanced the safety and reliability of fan case. 


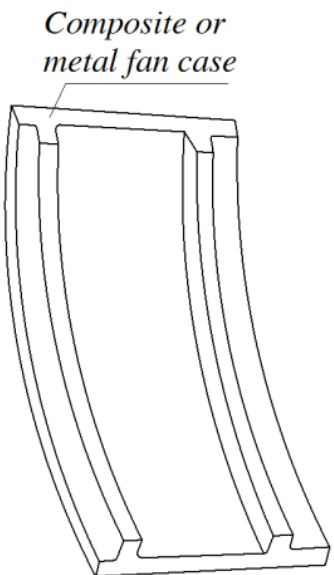

(a) Fan case

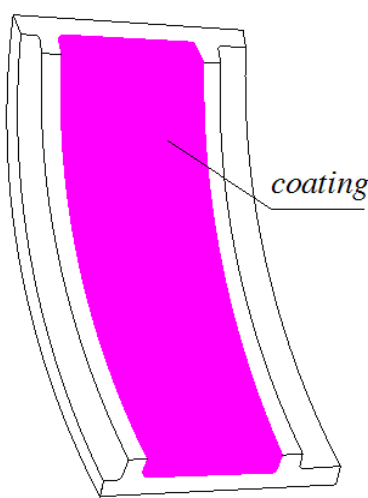

(b) Coating

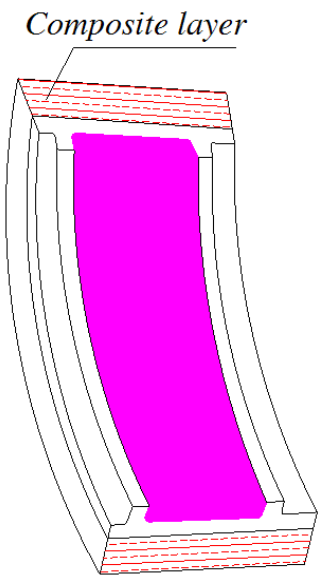

(c) Containment layer

Figure 9. Structure of containment layer

\section{Nitrogen Cooling System}

\subsection{Pipeline system}

Because of the high temperature in spool cavity, the tests for compressor and other components needed cooling nitrogen. The nitrogen cooling pipeline passed through the cavity of fan frame, and then came into the cavity of compressor spool, thereby nitrogen introducing to cool tester. Without changing the structure of fan frame cavity, the nitrogen cooling system was installed in test channel, as shown of Figure 1(7). The main pipe of cooling system was set in strut cavity, and the branch pipe passed through HUB MID BOX cavity, and then inserted into screw holes of NO.3 bearing seal, thereby nitrogen introducing to spool cavity, as shown in figure 10. After the end of the experiment, the cooling system could be disassembled, and the test cable should be assembled again. The assembly and disassembly process of the pipeline system had no influence on the structure of fan frame.

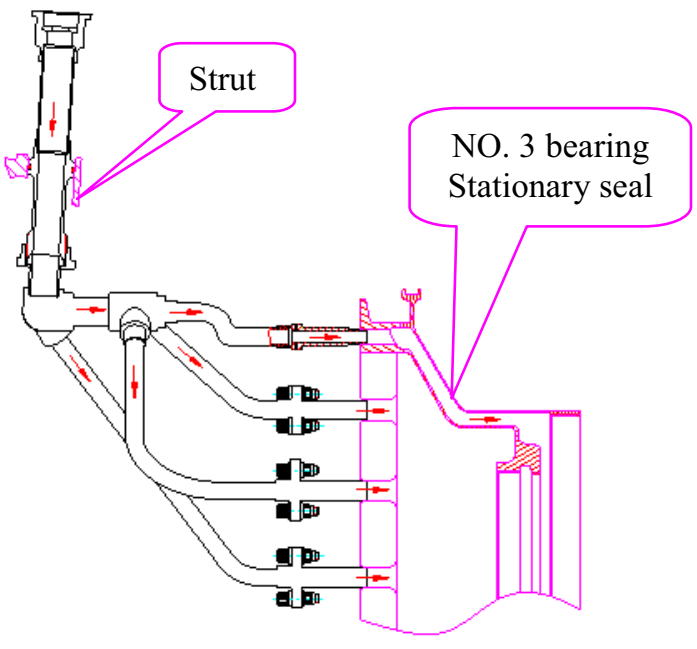

(a) Nitrogen cooling system

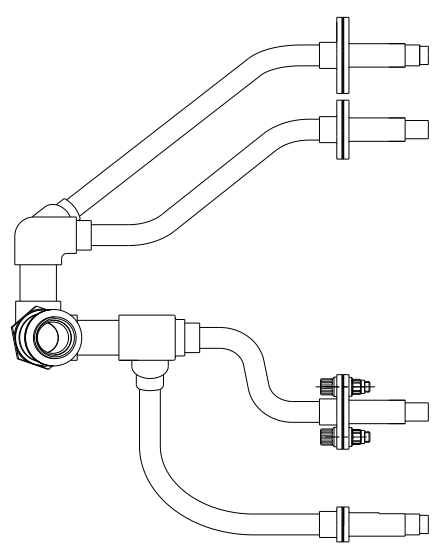

(b) pipeline system structure

Figure 10. Nitrogen cooling system in fan frame cavity 


\subsection{Seal and fixing structure}

Because of the limited space of the cavity in fan frame, there was no available support, so the sealing and fixing of pipeline systems was the difficulty in design. According to the air flow direction, in turn to use the cone seal, rubber ring seal, end face seal and plug seal structure, as shown in Figure 11. The cone seal structure was fixed with a sleeve nut, and solved the sealing and fixing problem simultaneously. The plug seal with small gap and gasket were used on frame interface, and the pipe joint is fixed with pressing broad. The pipe joint is sealed with rubber ring seal and end face seal. For reducing the aerodynamic loss, Pipe line routing is as short and straight as possible [8].

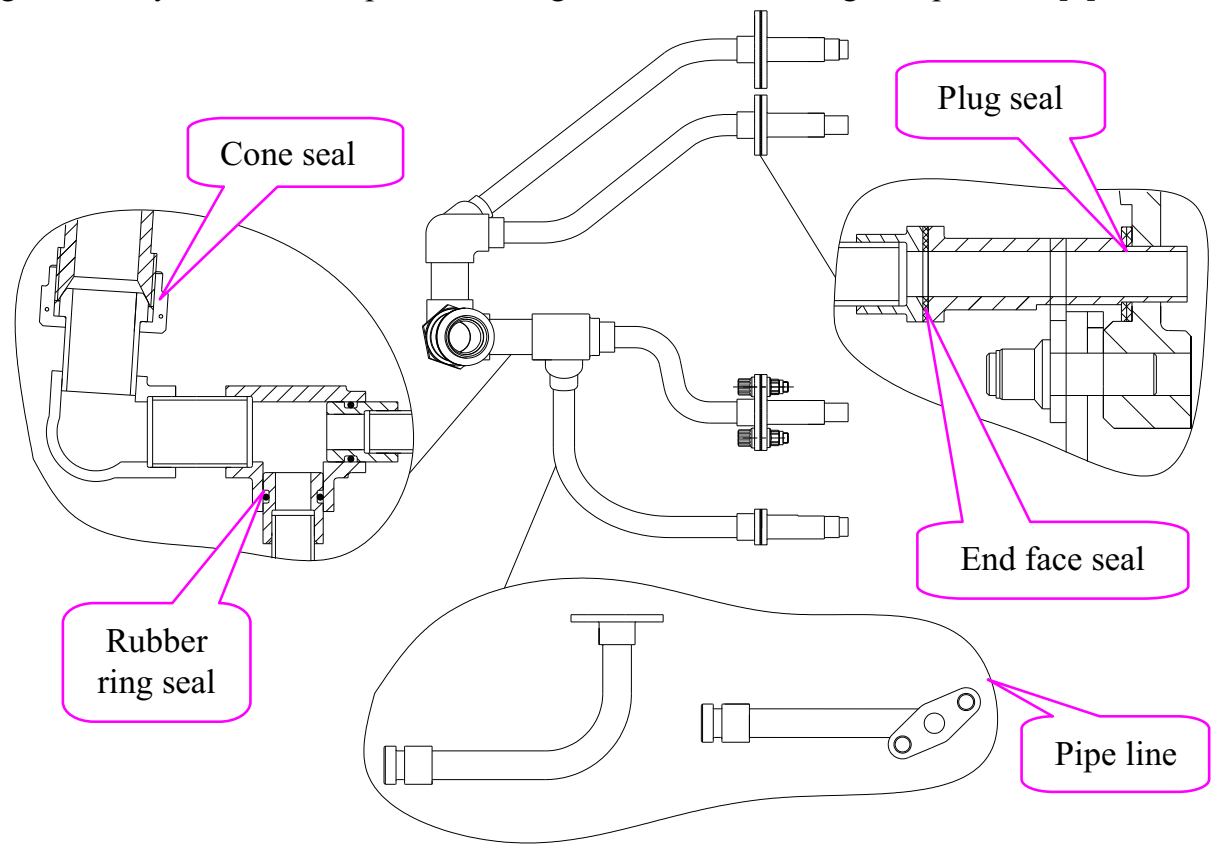

Figure 11. seal structure of pipeline system

\section{Conclusion}

The fan frame unit of high bypass ratio aero-engine had a larger design space. Using advanced composite could improve thrust-weight ratio, enhance structure performance, and reduce manufacturing cost of engine. The development trend of high bypass ratio aero-engine is super high bypass ratio, and the design technique of composite would become the bottleneck and restrict super high bypass ratio aero-engine. The application range of composite on aero-engine was gradually expanding, such as: fan blade, fan case, front core and thrust reverser.

With the increasing of bypass ratio, the inner cavity of fan frame was gradually enlarging, providing a design platform for more advanced pipeline system. In inner cavity of fan frame, the design of pipeline system could provide more testing projects for the core machine, so as to ensure the engine operating safely and stably. 


\section{References}

1. DU shan-yi, Advanced Composite Material Sand Aero Space Engineering, Acta Materiae Compositae sinica, 2007,24 (1)

2. HOU Peng, The Application of Acoustic Liner Technology on High Bypass Ratio Aero Engine, 15th Annual Meeting of China Association for Science and Technology, 2013

3. Kinsler,L.E.,Frey,A.R.,Coppens,A.B.,Sanders,J.V., Fundamentals of Acoustics Fourth Edition, John Wiley\&Sons,Inc.2000

4. M Perrino, J.E.S.G, Towards Development of an Active Single-layer Acoustic Liner for Jet Engine Noise Reduction, AIAA Aerospace Sciences Meeting Including the New Horizons Forum and Aerospace Exposition, 2013

5. TL Parrott, MG Jones, Status of duct liner technology for application to aircraft engine nacelles, Journal of the Acoustical society of America, 2005,118 (3)

6. $\mathrm{Z}$ Fan, $\mathrm{D}$ Gao, $\mathrm{T}$ Jiang, experimental study and numerical simulation of model casing containment, Journal of Nanjing University of Aeronautics \& Astronautics, 2006, 38 (5)

7. YU Ya-Bin, W Chen, Numerical Analysis of the Modeled Blade/Casing Containment, Journal of Aerospace Power, 2005, 20 (3)

8. BAI xiao-lan, Pipe Routing Algorithm for Aero-Engines, Machinery Design \& Manufacture, 2013,9 (1) 